\title{
In situ analysis of cadmium sulphide chemical bath deposition by an optical fibre monitor
}

\author{
B.R. Wakeling ${ }^{\text {a }}$, B. Degamber ${ }^{\text {a }}$, G. Kister ${ }^{\text {a }}$, D.W. Lane ${ }^{\text {a, } *}$, M.J. Bailey ${ }^{\text {b }}$, C. Jeynes ${ }^{\text {b }}$ \\ a Department of Engineering and Applied Science, Cranfield University, Shrivenham, Swindon, SN6 8LA, UK \\ b University of Surrey Ion Beam Centre, University of Surrey, Guildford, GU2 7XH, UK
}

\section{A R T I C L E I N F O}

Article history:

Received 5 August 2011

Received in revised form 26 June 2012

Accepted 2 July 2012

Available online 31 July 2012

\section{Keywords:}

Cadmium sulphide

Chemical bath deposition

In situ monitor

Optical fibre

\begin{abstract}
A B S T R A C T
The CdS window layer in thin film solar cells is frequently grown by chemical bath deposition (CBD). Deposited films are typically less than $100 \mathrm{~nm}$ thick and the inability to identify the exact start of the deposition can make $\mathrm{CBD}$ an imprecise process. This paper describes the construction and testing of a simple optical fibre sensor that detects the start of the deposition process and also allows for its mechanism to be studied. The in situ optical fibre monitoring technique utilises the change in optical reflectance off the glass/deposited film/precursor solution interfaces at an operating wavelength of $1550 \mathrm{~nm}$. A theoretical expression for the reflection of light from the interface is discussed and compared with experimental results. The monitoring technique shows the presence of two different deposition mechanisms. This result is confirmed by film densities calculated by Rutherford backscattering spectrometry and an optical model for ellipsometry measurements which indicates that the deposited CdS films consist of a double layer structure with a porous layer on top of a dense under layer. The application of the theoretical expression is optimised by assuming the refractive index of the CdS layer to be 2.02 . The ellipsometry model shows that the refractive index of the CdS deposited is 2.14 for a two layer model of the film that included a porous upper layer through the effective medium approximation.
\end{abstract}

(c) 2012 Elsevier B.V. All rights reserved.

\section{Introduction}

Chemical bath deposition (CBD) has been widely used to deposit cadmium sulphide (CdS) for photovoltaic applications because of its ability to reproducibly deposit uniform thin films. In CBD, where the chemical precursors are heated simultaneously, in order to allow greater control of the process it is important to determine the start temperature and time of the deposition in addition to the rate. This paper proposes a method of in situ monitoring using light reflected off the interface between an optical fibre and a precursor solution.

Two CBD reaction mechanisms for the formation of the CdS have been suggested, heterogeneous and homogeneous. Heterogeneous reactions are preferred for thin film solar cell formation because ion by ion deposition results in a dense CdS film structure [1]. Alternatively, the homogeneous reaction involves the formation of $\mathrm{CdS}$ particles or clusters within the solution, which are adsorbed onto the substrate. This causes the film to be powdery and non-adherent to the substrate [2]. The formation of particles in the solution has also been shown to reduce the final thickness of the deposited film and to increase the diffuse scatter of the film deposited [3].

Lincot and Borges identified three distinct stages of deposition with different growth rates [4]. Firstly, an induction time was reported where nucleation takes place on the substrate surface. A region of linear

\footnotetext{
* Corresponding author. Tel.: +44 1793 785226; fax: +44 1793785772.

E-mail address: d.w.lane@cranfield.ac.uk (D.W. Lane).
}

growth then occurs, where a compact layer is deposited, possibly where the heterogeneous growth mechanism dominates. The third and final region is associated with an increase in the growth rate due to a change in the dominant mechanism; this creates a porous layer possibly indicating a particle or cluster mechanism. This change in the mechanism shows that the chemistry in the solution changes as the reaction progresses. The relative period of each of these growth regions will ultimately determine the quality of the film produced.

Techniques do currently exist for the in situ measurement of the CBD growth rate, two examples being: quartz crystal microbalance and electrochemical impedance measurements [4] and monitoring the change in the solution transparency [5]. The limitations of these techniques are that they may require apparatus modification or that they have only been demonstrated for a limited range of film thicknesses. An in situ optical fibre monitoring technique for sputtered film has been developed for titanium and titanium oxide by Caranto et al. [6], who demonstrated the use of an optical fibre technique using an expression for a two interface (three medium) optical structure.

This work builds on other group's work, developing the in situ optical fibre technique for CBD growth methods. It aims to show that light reflected off the end of an optical fibre immersed in a CBD reaction solution is dependent on the optical properties of the interface at the end of the fibre. The small diameter of the fibre enables its implementation with minor modification to the apparatus and permits, due to the minimal loss of optical fibres, for analytical equipment to be stationed at a convenient distance from the experiment. 


\section{Experimental details}

The CdS CBD procedure of this work started with an initial solution of: thiourea $(0.057 \mathrm{M})$, ammonium chloride $(0.028 \mathrm{M})$ and cadmium chloride $(0.002 \mathrm{M})$. Ammonia was added to the solution to adjust its $\mathrm{pH}$ to 10.5 . CdS was deposited on sample substrates during each deposition. The sample substrates consisted of a glass with a transparent conducting oxide layer on one side. These were cleaned with acetone and detergent, loaded onto a glass mount within the deposition solution and rotated in the solution throughout the deposition process at a rate of $2 \mathrm{~Hz}$. The deposition solution with all of its constituents was heated to a temperature of $70 \pm 2{ }^{\circ} \mathrm{C}$ at a rate of $3{ }^{\circ} \mathrm{C} \mathrm{min}{ }^{-1}$. When the solution reached $70{ }^{\circ} \mathrm{C}$, an ultrasonic bath was used to agitate the solution, to minimise the formation of $\mathrm{CdS}$ particles during the deposition process [7]. Deposition progressed for $15 \mathrm{~min}$ to attain a film of $150 \mathrm{~nm}$. The reaction was stopped by removing the samples from the deposition solution and rinsing them with deionised water.

The in situ optical fibre measurements were made using SMF28 optical fibres (Corning, silica core diameter $6 \mu \mathrm{m}, 150 \mu \mathrm{m}$ with cladding included) operating at $1550 \mathrm{~nm}$. A $2 \times 2$ coupler was used to connect the Lumen Photonics (LPB-1550-D) broadband light source centred at $1550 \mathrm{~nm}$ and the Anritsu MN9710B optical spectrum analyser collecting the reflected light to the optical fibres, which passed into the deposition chamber via the coupler. Reflection peaks were monitored in the window 1540 to $1560 \mathrm{~nm}$. The fibre was stripped of its acrylate coating and the cladded end was cleaved and wiped with isopropanol before it was positioned within the deposition container close to the chamber walls. Collection of reflectance data was initiated at the point when all the reactant constituents had been added to the bath and heating began. The fibre was removed from the CBD solution at the same time as the sample substrates. The in situ measurements consisted of recording the intensity of the reflected signal with time at intervals of $12 \mathrm{~s}$. The intensity of the reflected light was corrected for the background measurements and recorded as the change of reflectance intensity, $\Delta \mathrm{R}(\mathrm{dB})$. This correction takes into account the difference in the quality of the cleaved surface between different depositions.

To investigate the deposition process, the films deposited on some of the optical fibres were examined using Rutherford backscattering spectrometry (RBS) and particle induced X-ray emission (PIXE). This employed the $2 \mathrm{MV}$ tandem accelerator at the University of Surrey Ion Beam Centre using a $1.557 \mathrm{MeV}^{4} \mathrm{He}^{+}$beam with a spot size of approximately $10 \mu \mathrm{m} \times 10 \mu \mathrm{m}$ and a beam current of $300 \mathrm{pA}$.

The physical thicknesses of the CdS films deposited on sample substrates were measured using an Alpha step 100 surface profiler. Optical properties were measured using a J.A. Woollam Co. M-2000UI rotating compensator ellipsometer in conjunction with CompleteEASE software (version 3.81), for the generation of optical models. A mean squared error (MSE) function was used to assess the quality of the fit of the optical models to the experimental data [8].

Infrared (IR) spectroscopy of the deposition reaction solution was carried out using thin walled vessels containing the solution loaded into a heating mount within a Nicolet (Magna IR 760) spectrometer. The spectrometer was setup to take a measurement every $30 \mathrm{~s}$ while the deposition solution was heated to $70{ }^{\circ} \mathrm{C}$ and then held at that temperature for $60 \mathrm{~min}$.

\section{Theoretical expression}

The reflection of light from the end of the optical fibre was modelled as the reflectance from the interface between three media (Fig. 1). The first medium is the optical fibre core, with a refractive index $\left(n_{0}\right)$ of 1.46; the second is the CdS film on the end of the fibre with a refractive index $\left(n_{1}\right)$ of 2.25 (at $1500 \mathrm{~nm} \mathrm{CdS}$ thin film [9]). The third is the solution which was approximated to water with a refractive index of 1.33 $\left(n_{2}\right)$. The CdS medium is assumed to have a homogeneous refractive

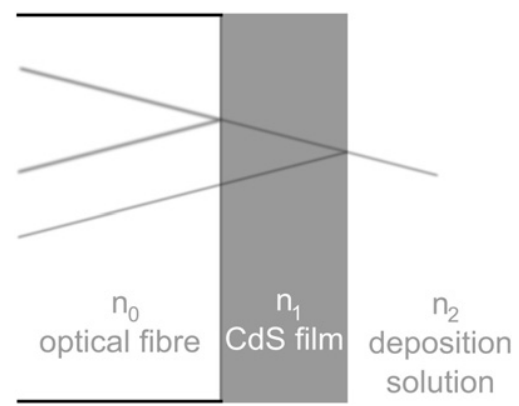

Fig. 1. The optical structure modelled for the calculated reflectance.

index and the solution optical properties are assumed to be constant during the deposition. The solutions' colour visually changes during the deposition; whether this is solely due to precipitates in the solution or not is unclear. The reflectance $R$ is given by Eqs. (1)-(3) [10].

$R=\frac{r_{1}^{2}+2 r_{1} r_{2} \cos 2 \delta+r_{2}^{2}}{1+2 r_{1} r_{2} \cos 2 \delta+r_{1}^{2} r_{2}^{2}}$.

\section{Where}

$r_{1}=\frac{n_{0}-n_{1}}{n_{0}+n_{1}}, r_{2}=\frac{n_{1}-n_{2}}{n_{1}+n_{2}}$

and

$\delta=\frac{2 \pi}{\lambda} n_{1} d_{1} \cos \psi_{1}$

where $\lambda$ is the wavelength of the light (1550 nm), $d_{1}$ is the thickness of the CdS film and $\psi_{1}$ is the angle of incidence between the normal to interface surface and the path of the light. The light path has been assumed to be normal to the interface and $\psi_{1}$ therefore equals to zero. The cone of acceptance for light to be transmitted along the optical fibre is $16.8^{\circ}$.

The change of reflectance $(\Delta R)$ for different thicknesses of $C d S$ film was calculated using the reflectance for when no CdS film was present as a reference (Fig. 2). A maximum reflectance occurs when the optical thickness $\left(t_{m}\right)$ is equal to one-quarter of the wavelength of the light (Eq. 4).

$t_{m}=\frac{\lambda}{4 n_{1}}=\frac{1550}{2.25 \times 4}=172.2 \mathrm{~nm}$

The calculations show that $\Delta \mathrm{R}$ is not very sensitive to small variations in the thickness of the middle medium, in the region 150 to $200 \mathrm{~nm}$. This indicates a possible limitation of the technique in monitoring the final thickness of the deposited films which are typically of this thickness.

\section{Optical measurements}

The results from the initial experiments clearly show a change in the intensity of the reflected signal with time (Fig. 3). The start of the reaction can easily be identified by the sharp increase in $\Delta R$, assumed to be associated with the first formation of CdS on the end of the fibre. The measured change in $\Delta \mathrm{R}$ differs from what would be expected from the theoretical model of $\Delta R$ (Fig. 2) if the film thickness varied linearly with time. The difference suggests that either the film thickness is not varying linearly with time or another parameter that affects the reflected light is varying during the reaction (for example the refractive index of the solution is changing with time). 


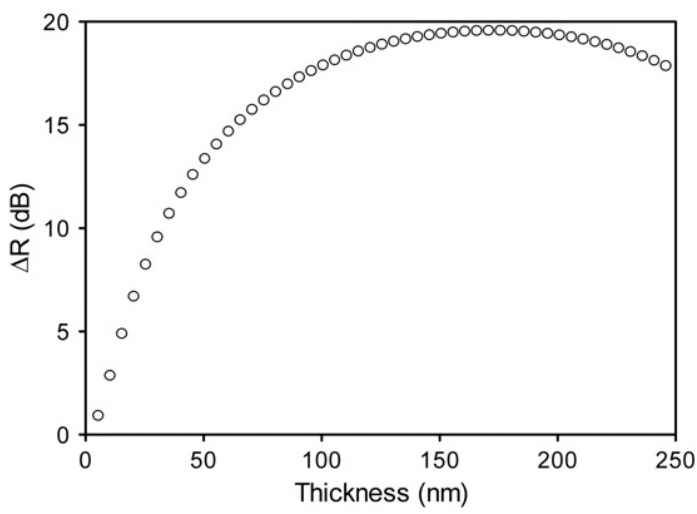

Fig. 2. The variation in the calculated $\Delta \mathrm{R}$ with film thickness.

The deposition time of the CBD was varied to produce a range of film thicknesses under constant deposition conditions. The thickness of the CdS deposited on the sample substrates was measured by profilometry and plotted against the largest change in the reflectance for each of the depositions outlined in Fig. 4. It is acknowledged that there may be a difference in the initial formation of the CdS on the end of a silica optical fibre and on the conducting layer on the sample substrates; this is discussed later. The calculated and measured values for $\Delta \mathrm{R}$ for different thicknesses of CdS films appeared to follow the same trend. However, the calculated data consistently had a higher $\Delta \mathrm{R}$ value than those values found experimentally.

It was proposed that the parameters used within the expressions (Eqs. 1-3) could be refined to produce a better fit to the data. In particular, consideration was given to the parameters relating to: the angle of the light $\left(\psi_{1}\right)$ at the interface at the end of the fibre and the refractive index of the CdS medium $\left(n_{1}\right)$. When the angle of incidence was set to $16.8^{\circ}$, instead of assuming the light was normal to the surface, the root mean square (RMS) value for the fit of the data to the expression changed from 2.76 to 1.09 . However with the incidence angle still set to $0^{\circ}$, the refractive index of the CdS layer was varied to fit the model to the data; the minimum RMS value of 0.98 was achieved when the refractive index of the $\mathrm{CdS}$ equalled 2.02 compared to its original set value of 2.25 .

This improvement in the RMS may be linked with the CdS layer having a less dense structure, such as the double layer structure described by Lincot and Borges [4] and the change in the optical constant represents a better approximation of the properties of the double layer structure. Although changing both parameters was found to improve the agreement between the model and the data, the relevance of the different parameter values on the physical system was not determined.

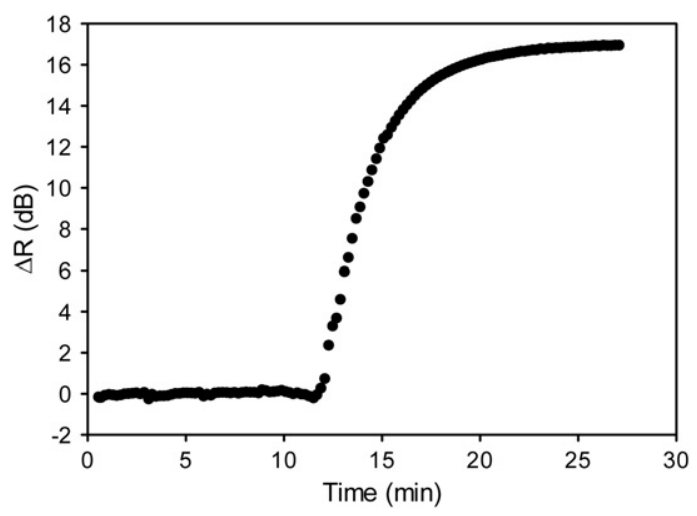

Fig. 3. The change in $\triangle \mathrm{R}$ for light reflected from the end of an optical fibre during a CBD.

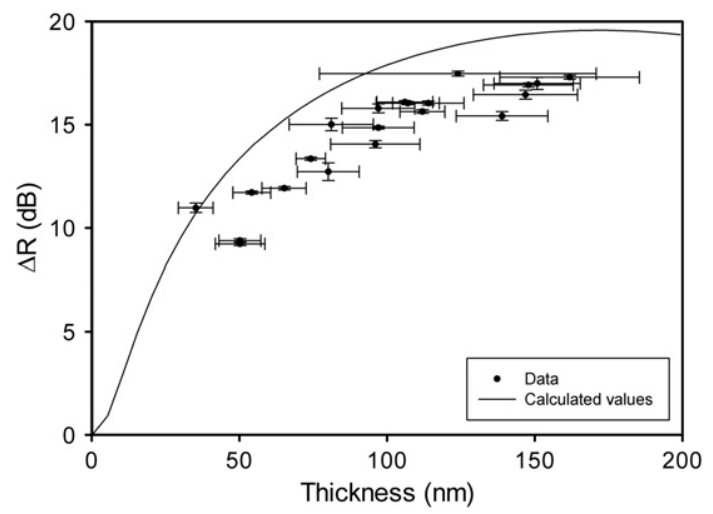

Fig. 4. The $\Delta R$ at the end of the deposition is plotted against the thickness of the CdS deposited on the substrate samples. The calculated values are included for comparison.

With no justification for a certain set of parameter values, the originally assumed values have been used in the rest of this work.

The expressions (Eqs. 1-3) were used to approximate the thickness of the CdS layer from the reflectance intensity as a function of deposition time (Fig. 5A). The initial $300 \mathrm{~s}$ of deposition is examined in more detail in Fig. 5B where both the $d_{1}$ and $d_{1}^{2}$ are plotted against the deposition time. This clearly shows that this initial region is well represented by a constant deposition rate and therefore a reaction rate limited deposition, as opposed to a diffusion limited deposition. This is in agreement with the results reported by Lincot and Borges [4]. The deposition rates observed here imply that two deposition mechanisms are dominant at different times during the longer depositions. The ultrasonic bath used throughout the growth period was intended to minimise the adsorption
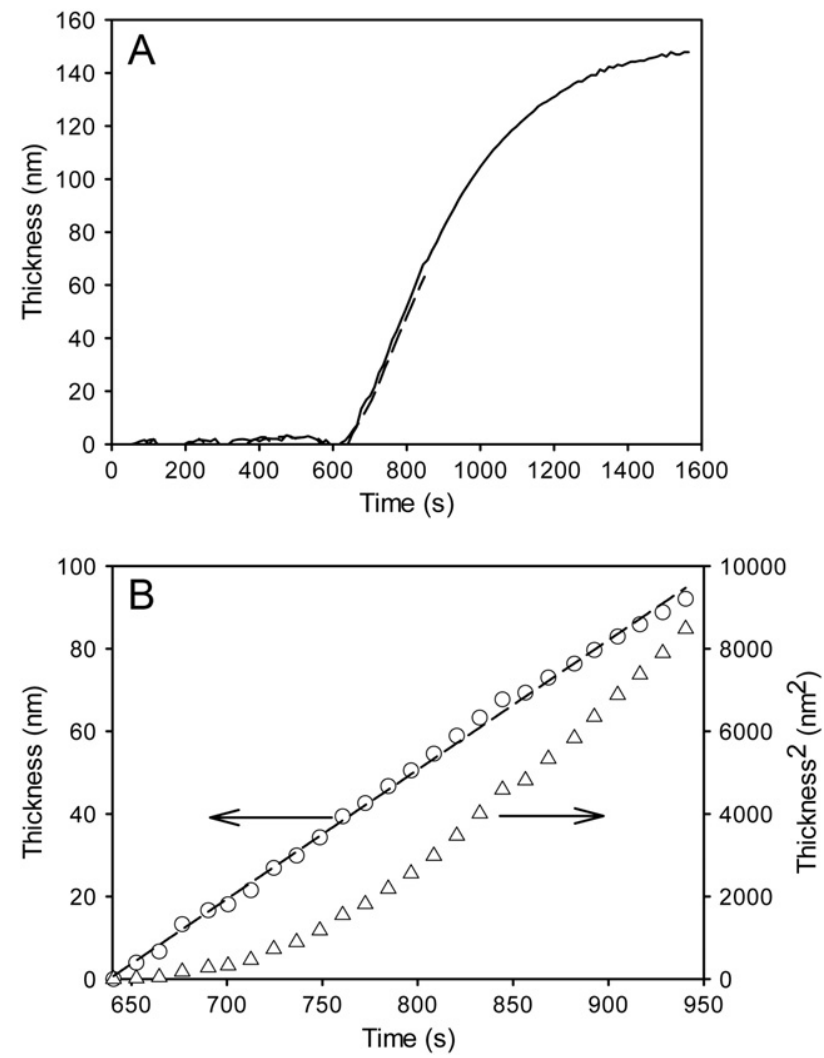

Fig. 5. (A) The change in the approximate thickness of the film with time calculated from the change in reflectance, for two deposition runs (final thicknesses of the layers on the glass substrates were $148 \pm 15.3 \mathrm{~nm}$ (solid line) and $65 \pm 7.5 \mathrm{~nm}$ (broken line)). (B) Examination of the first $300 \mathrm{~s}$ growth for the $148 \mathrm{~nm}$ thick film, broken line linear regression to data. 


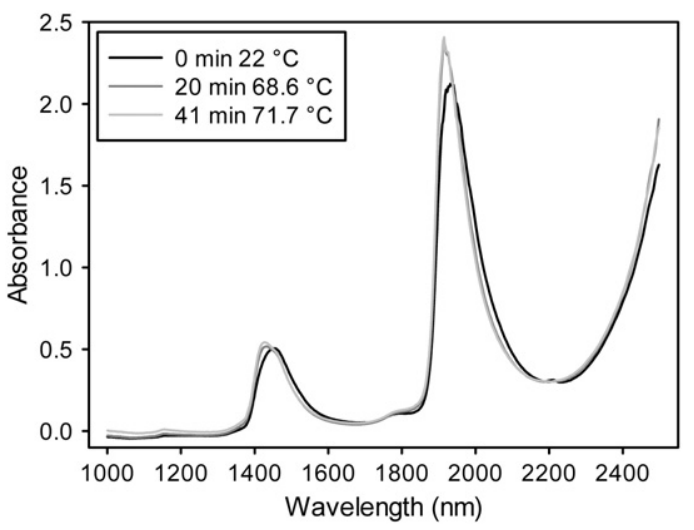

Fig. 6. The near IR absorbance spectra of a small amount of the reaction solution as it was heated to and then held at $70{ }^{\circ} \mathrm{C}$. The wavelength of the light used in the optical fibre measurements was $1550 \mathrm{~nm}$.

of large particle clusters during deposition. However, two different deposition mechanisms were observed to dominate at different times during growth, indicating that heterogeneous adsorption had not been prevented.

\section{Solution spectroscopy and RBS measurements}

The optical model described in the previous section assumed the refractive index of each material to remain constant during film growth. However, as the film is deposited, the bath solution becomes depleted and its optical properties may change. A selection of the spectra collected by an IR spectrometer at different times during the measurement can be seen in Fig. 6.

The spectra show the same structure as that for pure water previously published by Jensen et al. [11], with two strong absorbance bands at 1450 and $1940 \mathrm{~nm}$ that correspond to water combination bands. This study showed only a small change in absorbance as temperature increased from 25 to $42^{\circ} \mathrm{C}$. Similarly, our results show only a small change over the period of our measurements, corresponding to temperatures between 22 and $72{ }^{\circ} \mathrm{C}$. As the solution is heated, the energy of the bonds within the material changes and this would cause a small shift in the absorbance peaks. The results indicate a small variation in the absorbance during film growth and support the simplifying assumption of a constant refractive index for the solution.

The thickness of the deposited CdS on the end of the optical fibre has been assumed to be the same as the CdS deposited on the sample substrates. RBS and PIXE measurements at Surrey University were performed to investigate the deposited material on the end of six optical fibres. Scanning electron microscopy was used to examine the ends of the fibres and it showed that the material appeared to be uniformly covering the $6 \mu \mathrm{m}$ cores in the middle of the fibres.

The RBS results indicate the deposited CdS to be not stoichiometric with the films on the fibres being $\mathrm{Cd}$ rich, see Table 1 . The uncertainty in the stoichiometry is mainly due to the accuracy with which the sulphur component of the data could be fitted. The calculated densities of the films decrease as their thicknesses increase, with the exception of the thinnest film. This result would support the idea of a change in the dominant mechanism during the deposition, with less dense material being deposited later in the deposition. The PIXE analysis showed the presence of chlorine within the films and this combined with lower density, may corroborate the nonstoichiometry of the films. A limitation of this analysis is the assumption that the same thickness of film is present on both the sample substrates and the ends of the optical fibre. The different materials of the fibres and the substrates (silica compared to transparent conducting oxide) may cause a different nucleation stage to occur, ultimately affecting the final achievable material thickness.

\section{Ellipsometry measurements}

Individual layers of CdS were grown on the silica substrates to provide simple, controlled structures for ellipsometry measurements. Work by Ninomiya and Adachi [12] produced a detailed oscillator model for single crystal CdS based on the energy band structure of wurtzite CdS. This model was used as a guide in this study to create a model for polycrystalline material. For an as-deposited CdS layer, three oscillator functions were used to model the optical constants: a Cody Lorentz oscillator [8] and two Lorentzian oscillators.

The single layer of as-deposited CdS was initially modelled by a single layer within the ellipsometry model. However, this model did not produce a good match to the experimental data, with an MSE of 25.87. A second model, with two layers representing the CdS film produced a very good match to the data, with a MSE of 3.44 (Fig. 7). The first layer was $84.3 \mathrm{~nm}$ thick and comprised the three oscillators which modelled the broad optical constants of CdS. A second layer ( $46.4 \mathrm{~nm}$ thick), combined the properties of the first CdS layer with those of a "void" material $(\mathrm{n}=1, \mathrm{k}=0)$ in an effective medium approximation layer, using a Bruggeman function. The inclusion of the second layer indicated a void fraction of approximately $74 \%$ and improved the quality of the fit around and above the band gap. The refractive index found for the CdS within the model, at $1550 \mathrm{~nm}$ was 2.14 , notably less than the published value of 2.25 for polycrystalline $\mathrm{CdS}$ at this wavelength [9]. The stability of the fit compared with other models with more layers gave confidence in the two layer model result. The second layer indicates the presence of a less dense layer of CdS on top of CdS. This agrees with the CBD mechanism proposed in the literature by Lincot and Borges [4]. The two layer model agrees with work by Sandoval-Paz et al. [13], who used spectroscopic ellipsometry to propose properties of the microstructure of the CdS layer deposited by CBD.

\section{Conclusions}

An optical fibre in situ monitoring technique has been shown to be a viable way of monitoring a $\mathrm{CBD}$. The technique is sensitive to the start of the deposition, with its sensitivity reducing as the thickness of the deposited CdS film approaches the quarter wavelength of $172.5 \mathrm{~nm}$. A simple expression for the reflectance [6] has been used in this work, and the limitations of the parameter values used have also been assessed. The homogenous single layer of CdS representation has been

Table 1

The Cd rich Cd:S ratios and calculated densities measured by RBS, for the films deposited on the end of six optical fibres.

\begin{tabular}{|c|c|c|c|c|c|c|}
\hline \multirow[b]{2}{*}{$\begin{array}{l}\text { Thickness of films on sample substrates measured } \\
\text { by Alpha step (nm) }\end{array}$} & \multicolumn{6}{|c|}{ Six different optical fibres and their corresponding samples } \\
\hline & $54 \pm 6$ & $80 \pm 11$ & $98 \pm 12$ & $112 \pm 8$ & $147 \pm 18$ & $148 \pm 15$ \\
\hline $\begin{array}{l}\text { RBS determined Cd:S ratio of the optical fibre films } \\
\text { (error } 10 \% \text { or less) }\end{array}$ & 1.39 & 1.32 & 1.14 & 1.40 & 1.30 & 1.26 \\
\hline Calculated densities of the optical fibre films $\left(\mathrm{g} \mathrm{cm}^{-3}\right)$ & $1.64 \pm 0.18$ & $2.49 \pm 0.34$ & $2.53 \pm 0.31$ & $2.33 \pm 0.17$ & $2.04 \pm 0.25$ & $2.01 \pm 0.20$ \\
\hline Mean density $\left(\mathrm{g} \mathrm{cm}^{-3}\right)$ & $2.17 \pm 0.62$ & & & & & \\
\hline
\end{tabular}



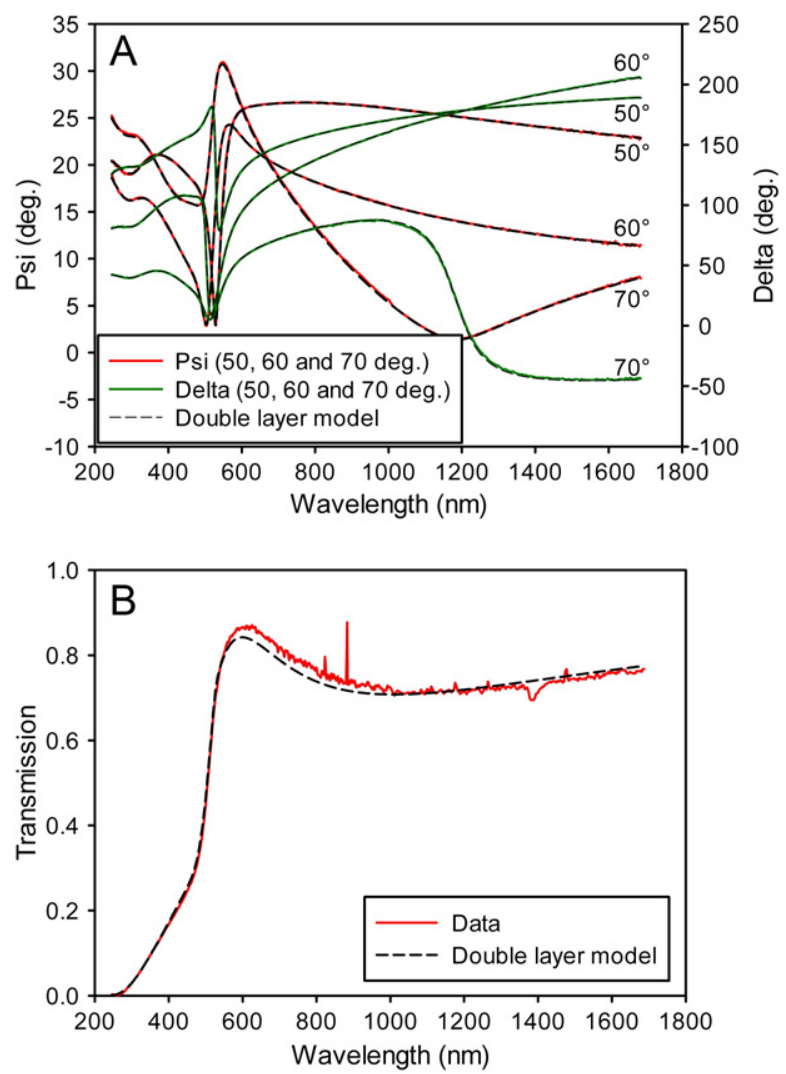

Fig. 7. Ellipsometry data for as-deposited CdS on a silica substrate, modelled with a double layer. The figure shows experimental data (solid line) measured in (A) reflection and (B) transmission modes, and the modelled data (broken line) fitted the data with an overall MSE of 3.44 .

shown to contribute towards the inaccuracy in the calculated reflectance. The refractive index of the single CdS layer could be changed to 2.02 to improve the fit; however the ellipsometry analysis showed a double layer to be a more accurate model of the film, with a CdS refractive index of 2.14. This had an upper layer with a high void fraction (approximately 75\%) and, when combined with the CdS refractive index being lower than that expected for polycrystalline CdS, was consistent with the low average film density observed by RBS.

Monitoring CdS depositions showed the presence of two growth mechanisms for thicker films, in agreement with Lincot and Borges [4]. The decrease in the density of the films deposited with increasing thickness and the strong results of the ellipsometry model, confirm the presence of two mechanisms dominating at different stages during the deposition.

The monitoring technique presented in this paper could be readily incorporated into an existing deposition system and could assist in the growth of high quality thin films through accurately detecting the start of film growth. In addition, CBD generates large quantities of Cd waste, the fibre optic sensor should help minimise this by more carefully controlling deposition.

\section{Acknowledgment}

This work was funded by the Research Councils UK Energy Programme as part of the SUPERGEN Consortium, Photovoltaic Materials for the 21st Century (EP/F029624/1).

\section{References}

[1] R.O. Borges, D. Lincot, J. Electrochem. Soc. 140 (1993) 3464.

[2] T.L. Chu, S.S. Chu, Solid-State Electron. 38 (1995) 533.

[3] I.O. Oladeji, L. Chow, J. Electrochem. Soc. 144 (1997) 2342.

[4] D. Lincot, R.O. Borges, J. Electrochem. Soc. 139 (1992) 1880.

[5] R. Saez-Araoz, D. Abou-Ras, T.P. Niesen, A. Neisser, K. Wilchelmi, M.C. Lux-Steiner, A. Ennaoui, Thin Solid Films 517 (2009) 2300

[6] N.R.Y. Caranto, S.C. Kaddu, J. Szajman, M.M. Murphy, S.F. Collins, D.J. Booth, Meas. Sci. Technol. 4 (1993) 865.

[7] U. Ketipearachchi, D.W. Lane, K.D. Rogers, J.D. Painter, M.A. Cousins, Mater. Res. Soc. Symp. Proc. 836 (2005) 161.

[8] J. Orava, T. Wagner, J. Sik, J. Prikryl, M. Frumar, L. Benes, J. Appl. Phys. 104 (2008) 043523.

[9] E.D. Palik, Handbook of Optical Constants of Solids, Academic Press, New York, 1997.

[10] O. Heavens, Optical Properties of Thin Solid Films, Butterworths, London, 1955. Section 4.8.

[11] P.S. Jensen, J. Bak, S. Andersson-Engels, Appl. Spectrosc. 57 (2003) 28.

[12] S. Ninomiya, S. Adachi, J. Appl. Phys. 78 (1995) 1183.

[13] M.G. Sandoval-Paz, M. Sotelo-Lerma, A. Mendoza-Galvan, R. Ramirez-Bon, Thin Solid Films 515 (2007) 3356. 